\title{
Estimación del peligro de inundaciones: actores y respuestas sociales frente al escenario de riesgo en los barrios del sur de Mar del Plata, Buenos Aires (Argentina)
}

\section{Flood hazard estimation: actors and social responses to the risk scenario in the southern neighborhoods of Mar del Plata, Buenos Aires (Argentina)}

\begin{abstract}
Mario Alejandro Maya
Doctorando en Geografía por la Universidad Nacional del Sur (UNS). Becario Doctoral en el Consejo Nacional de Investigaciones Científicas y Técnicas (CONICET). Centro de Investigaciones Geográficas y Socio-Ambientales. Facultad de Humanidades. Universidad Nacional de Mar del Plata. Funes 3350 (7600) Mar del Plata, Buenos Aires, Argentina, mayamarioalejandro@gmail.com.mx
\end{abstract}

Recibido: 21 de junio 2019 || Aprobado: 17 de febrero 2020

\section{Resumen}

En los últimos años, el sur de Mar del Plata ha sido testigo del crecimiento de urbanizaciones cerradas. Al respecto, se realizaron inversiones en la construcción y equipamiento específico que han modificado la geografía del lugar. Sumado a ello, en dicho sector de la ciudad se registró un aumento en el número de inundaciones y anegaciones. En consecuencia, se produjeron grandes pérdidas materiales y serios condicionamientos para el normal desenvolvimiento de la comunidad. Ante esta problemática, el objetivo principal del trabajo es realizar un diagnóstico sobre los riesgos que probables eventos peligrosos desarrollados en cercanías a los emprendimientos privados provoquen en el Barrio Parque Independencia. Así, el trabajo presenta una doble finalidad: por un lado, considerar para la problemática el modo en que se han articulado actores de distinto nivel de organización y rol; $y$, por el otro, realizar un diagnóstico del área de estudio con el fin de estimar el escenario de riesgo al que están expuestos los habitantes ante las probables pérdidas directas e indirectas que causaría una inundación sobre el territorio. El texto concluye que, como resultado del patrón de ocupación territorial y las componentes de vulnerabilidad identificadas en los contextos socio-económicos expuestos al peligro, sustenta la caracterización de dicho territorio como un escenario de riesgo.

Palabras clave: Urbanizaciones cerradas; Escenario de riesgo; Actores sociales; Peligrosidad

Abstract

In recent years, southern Mar del Plata has witnessed the growth of gated community developments. In this regard, investments were made in the construction and specific equipment that have modified the geography of the place. In addition to this, there was an increase in the number of floods in that city sector. Consequently, there were large material losses and serious conditioning for the normal development of the community. Given this problem, the main objective of the work is to make a diagnosis of the risks that probable "dangerous events" developed in close proximity to private enterprises cause in the Parque

Cita sugerida: Maya, M.A. (2020). Estimación del peligro de inundaciones: actores y respuestas sociales frente al escenario de riesgo en los barrios del sur de Mar del Plata, Buenos Aires (Argentina). Estudios Socioterritoriales. Revista de Geografía, (27), 040. DOI: https://doi.org/10.37838/unicen/est.27-040 
Independencia neighborhood. Thus, the work has a double purpose: on the one hand, considering the way in which actors from different levels of organization and role have been articulated; and, on the other hand, making a diagnosis of the study area in order to estimate the risk scenario where the inhabitants are exposed to the probable direct and indirect losses that would cause a flood over the territory The text concludes that, as a result of the pattern of territorial occupation and the components of vulnerability identified in the socio-economic contexts exposed to the danger, it supports the characterization of said territory as a risk scenario.

Keywords: Gated community; Risk scenario; Social actors; Dangerousness 


\section{INTRODUCCIÓN}

Las urbanizaciones cerradas, ubicadas en áreas periféricas y destinadas a las clases privilegiadas son producidas por los desarrolladores urbanos, actor social que invierte en tierras periurbanas baldías y las comercializa, previo acondicionamiento de la infraestructura urbana y de las características ambientales. La concentración de la población en paisajes urbanos dominados por la tecnología y la infraestructura está en aumento a nivel nacional y regional. Es una tendencia que no solo se desarrolla en las grandes áreas metropolitanas, sino que su lógica se traslada hacia ciudades intermedias como La Plata, Bahía Blanca y, en este caso, Mar del Plata.

Considerando que el desarrollo urbano es impulsado por la actividad humana, la mayor parte de los cambios en el territorio que se dan en y alrededor de las ciudades son intencionales. De forma que la producción del espacio urbano y la permanencia de estructuras físicas se asocian a los procesos socio-económicos que les dieron origen. Así, se pone en discusión la lógica con que la sociedad incorporó diferentes áreas al proceso de urbanización. No obstante, la construcción de emprendimientos urbanos cerrados en el sector sur de la ciudad de Mar del Plata ha generado una serie de problemas en los barrios adyacentes. En este marco, la variable climática ejerce su influencia sobre el territorio. La eventual sucesión de precipitaciones y su mayor intensidad tiende a provocar anegaciones e inundaciones que inciden en el normal desenvolvimiento de los habitantes. Como sostienen Balvanera y Cotler, el balance es complejo: se ha privilegiado la posibilidad de obtener ciertos tipos de beneficios a costa de otros; se han favorecido los satisfactores a corto plazo a costa de aquellos a mediano y largo plazo; se ha puesto énfasis en la obtención de bienes en el entorno inmediato a costa de consecuencias en todo el ambiente (Balvanera y Cotler, 2007).

\section{MARCO TEÓRICO}

El análisis urbano-rural de las áreas en expansión basado en el modelo tradicional de oposición campo-ciudad no alcanza para comprender los procesos que se dan en ellas. Su estudio requiere de enfoques capaces de asumir la interacción urbano-rural que define la zona de interfase. El marco teórico-metodológico de los sistemas complejos ofrece una perspectiva interesante para analizar e interpretar gran parte de los procesos que tienen lugar en el periurbano (Zulaica, Ferraro y Echechuri, 2010). La complejidad inherente a cualquier situación de riesgo, impone la necesidad de indagar bajo una visión holística las relaciones e interdependencias entre las amenazas (o peligros), los contextos expuestos vulnerables y las respuestas de los actores sociales (Monti, 2013). García (2006) sintetiza como sistema socioambiental complejo, a aquel donde se hacen manifiestas las relaciones, funciones y mutuas dependencias entre atributos y procesos del medio socio-económico y político, con elementos y fenómenos propios del medio bio-físico-natural, en su función de soporte y condicionante del desarrollo territorial. A su vez, destaca que es una totalidad emergente derivada de un ecosistema natural transformado por el hombre.

El crecimiento de la ciudad de Mar del Plata se ha dado de manera espontánea y desordenada, cuyo resultado es un territorio periurbano fragmentado, con escasa consolidación y vulnerable a ciertos peligros como las inundaciones. Las políticas de planificación urbana no han guiado ese incremento. Fernández et al. (1999), en un estudio sobre la sustentabilidad natural marplatense, indican que las problemáticas originadas por las diferentes prácticas urbanas de gestión impactan sobre el capital natural (recursos), base de sustentación de la ciudad, e inciden sobre las condiciones de habitabilidad social, principalmente de la periferia y de la interfase urbano-rural en la que avanza el crecimiento urbano.

Las condiciones de habitabilidad cambian a partir del impacto que dichos procesos tienen en el territorio. Por ejemplo, en el barrio Parque Independencia se ponen de manifiesto en la vulnerabilidad de su población, reflejado en daños en 
las viviendas y locales comerciales, evacuados y asistencia de personas, cortes en la energía eléctrica, suspensión de clases, daños en la infraestructura vial y equipamiento urbano. Es decir, el concepto de vulnerabilidad ayuda a determinar al espacio como "susceptible o no a sufrir un daño o perjuicio ante un eventual fenómeno natural" (Wilches-Chaux, 1993, p. 43). De esta interpretación, Saurí Puyol (2003) añade dos dimensiones más a la exposición física que son respectivamente: a) la resistencia o la capacidad del sistema socionatural de seguir su funcionamiento normal a pesar de haber sufrido una perturbación; y b) la resiliencia o la capacidad de recobrarse de las alteraciones inducidas por una perturbación que han afectado sustancialmente el funcionamiento de este sistema. Estas definiciones permiten obtener una mayor precisión en las características de la población que se encuentra expuesta a fenómenos de riesgo. Estos eventos generalmente generan escenario de desastre frente a las inundaciones extremas, en las que las lluvias superan la capacidad material de sectores de la población para absorber, amortiguar o evitar los efectos de este acontecimiento $y$, por ende, interrumpe la actividad socioeconómica de una comunidad y produce un cierto daño directo e indirecto (Herzer, 1990).

A diferencia de las urbanizaciones cerradas que la limitan, las viviendas del área objeto de estudio presentan riesgo de inundación y consecuentemente probabilidad de registrar pérdidas materiales e, incluso, humanas. Durante un evento climático en abril de 2017, Rodrigo Goncálvez, director de Defensa Civil del municipio, señaló que "más de 100 personas fueron evacuadas y muchas están a la espera de que se les recompongan sus domicilios y puedan volver a sus hogares" (Zona 54, 09/04/2017). El riesgo es un concepto extraño y complejo, representa algo que parece irreal, relacionado con el azar, con posibilidades, con algo que aún no ha sucedido (Cardona, 2001). Por lo tanto, el autor lo define como un "daño esperado ante la probabilidad de ocurrencia de eventos peligrosos y de la vulnerabilidad de los elementos expuestos en un cierto sitio y en un cierto periodo de tiempo" (Cardona, 1993, p. 45).

De acuerdo a la afirmación precedente, el espacio que se aborda presenta un nivel de riesgo aceptable (Maskrey, 1993) ya que la comunidad afectada está dispuesta a asumir determinadas consecuencias a cambio de una tasa de beneficios que no desean relegar. En este contexto de incertidumbre, la peligrosidad, entendida como la "probabilidad de que una zona dada la superficie terrestre se vea afectada por un determinado proceso, la cual puede ser potencialmente peligrosa para el hombre (Cendrero y Fischer, 1997), está latente si se analizan los registros climáticos de la ciudad, en especial los periodos mensuales de precipitaciones. El desarrollo de un desastre es probable, pero en estos contextos de alta vulnerabilidad la posibilidad de daños, por mínimos que sean, genera un impacto significante en los habitantes del lugar. Lavell define el concepto de desastre como

la situación o proceso social que se desencadena
como resultado de la manifestación de un fenó-
meno de origen natural, tecnológico o provoca-
do por el hombre que, al encontrar condiciones
propicias de vulnerabilidad en una población,
causa alteraciones intensas, graves y extendidas
en las condiciones normales de funcionamiento
de la comunidad. (2013, p. 18$)$

De este modo, se observan dos situaciones: la peligrosidad de un evento natural y la vulnerabilidad de habitantes y elementos de infraestructura dispuestos en el lugar. Según Cardona (1993, p. 54), la exposición de los elementos y configuraciones espaciales se vincula con el "contexto social, material y ambiental representando por las personas, y por los recursos y servicios que pueden verse afectados por la ocurrencia de un evento de peligro". Además, el autor incluye, en la anterior definición, las actividades humanas, así como "todos los sistemas realizados por el hombre tales como edificaciones, líneas vitales e infraestructura, centros de producción, utilidades, servicios, la gente que los utiliza, y el medio ambiente" (Cardona, 1993, p. 54). En este sentido, el análisis de riesgo, es

el resultado de relacionar la amenaza y la vulnera- 
bilidad de los elementos expuestos, con el fin de determinar los posibles efectos y consecuencias sociales, económicas y ambientales asociadas a uno o varios fenómenos peligrosos. Cambios en uno o más de estos parámetros modifican el riesgo en sí mismo, es decir, el total de pérdidas esperadas y consecuencias en un área determinada. (Lavell, 2013, p. 17)

Por último, es imprescindible destacar que los desastres no son naturales. La misma se caracteriza por su vinculación con las actividades humanas, en nuestro caso: la construcción y/o modificación del terreno para la edificación de emprendimientos de barrios privados. Al mismo tiempo, se asocia a variables naturales interpretadas y recalificadas por el hombre, lo cual vuelve aún más significativa la connotación negativa en la población por su potencialidad de daño. De manera que el hombre se encuentra comprometido en el plano material, el simbólico -valores culturales y morales- $y$ en los diversos componentes del ecosistema bio-geo-físico que lo hacen vulnerables a determinados peligros, configurando en consecuencia escenarios de riesgo. Monti (2007) define a los mismos como la coincidencia espacial de procesos que por su magnitud, frecuencia $e$ intensidad constituye un factor de peligrosidad desde la perspectiva humana y contextos físicos, socioeconómicos y ecológicos con distintas condiciones de vulnerabilidad frente a determinados peligros.

La importancia de evaluar la peligrosidad yace en la necesidad de construir una visión objetiva y real de la potencialidad de una población. Por este motivo, el tipo de conocimiento a construir apela a una matriz epistemológica holística y a la vez precisa del fenómeno, sea este de origen natural, tecnológico o mixto. De este modo, será posible fomentar respuestas adecuadas y efectivas de la población frente al peligro $y$, a su vez, permite establecer la/s fuente/s origen de la peligrosidad y sus posibles cadenas causales.

A partir del presente trabajo y, aplicando los conceptos de Simioni (2003) y Sorensen, Mc Creary, y Brandani (1992), se pretende realizar una clasificación de actores del riesgo que permita obtener una mayor comprensión de las lógicas e intereses que rodean a cada uno de los actores involucrados. Según ambos autores, podría caracterizarse a los mismos de dos maneras: los actores estructurales, que se caracterizan por hallarse ligados directamente a la evolución y el desenlace del fenómeno; los actores funcionales son aquellos cuyo rol fundamental es servir de nexo entre los ámbitos y los actores estructurales. La clasificación de actores sociales del riesgo permite obtener una mayor comprensión de las lógicas e intereses que rodean a cada uno de los actores involucrados en la problemática.

\section{MÉTOdOS}

El enfoque metodológico para desarrollar la investigación es mixto. La diversidad de fuentes seleccionadas impone la utilización de distintas técnicas. Ello implica el empleo de diferentes metodologías de recopilación y análisis de la información que incluyen tanto el examen cuantitativo como el tratamiento cualitativo de las mismas.

Para llevar adelante la investigación, se toman como referencia los mayores emprendimientos privados (Rumencó, Arenas del Sur, Arenas Chico y Las Prunas) y uno de sus barrios adyacentes. La zona elegida para su análisis se encuentra localizada en el periurbano sur de Mar del Plata y comprende el barrio Parque Independencia.

Se utilizó el enfoque cuantitativo para la obtención y el tratamiento de datos secundarios provenientes de fuentes oficiales como la Encuesta Permanente de Hogares y del Censo Nacional de Población 2010, de instituciones públicas nacionales (Obras Sanitarias Sociedad del Estado -OSSE-, Instituto Nacional de Tecnología Agropecuaria -INTA-, Servicio Meteorológico Nacional -SMN-, entre otros) y otros procedentes de distintas secretarías del Municipio de General Pueyrredón. También se incorporaron al análisis los datos primarios recogidos de 65 encuestas cerradas, hechas con carácter aleatorio a los individuos del barrio 
Parque Independencia. En las mismas se dio especial énfasis a la obtención de datos relacionados con las características socioeconómicas de los habitantes y los atributos de las viviendas del lugar.

A su vez, se realizó un procedimiento cualitativo para abordar la realidad subjetiva de la población. De este modo, se han realizado entrevistas a informantes calificados (presidentes de sociedades de fomento, directores de las instituciones educativas, encargados de distintos comedores y merenderos, entre otros) para profundizar aún más en la cuestión trabajada. No obstante, sumado a lo anteriormente dicho, hay que destacar los aportes que la observación directa y el relevamiento de campo aportaron a la realización del trabajo. Todos ellos son datos cualitativos que permiten contextualizar los fenómenos y lograr una mayor profundidad en la problemática. Los métodos de campo empleados incluyeron visitas frecuentes para realizar observaciones directas sobre los atributos del sistema físico-natural y del sistema socioeconómico que caracteriza el sitio. Por último, se utilizaron fuentes bibliográficas específicas, ordenanzas municipales, leyes de ordenamiento territorial, artículos periodísticos, entre otras, que permiten avanzar en el análisis de los discursos y prácticas de los grupos sociales.

\section{ÁREA DE ESTUDIO}

El área seleccionada para el trabajo se extiende fuera de los límites del ejido urbano, en el área periurbana sur de la ciudad de Mar del Plata, provincia de Buenos Aires. La misma se ubicada a 4 kilómetros de la zona litoral sur, aproximadamente.

El barrio Parque Independencia -área referencial del presente estudio- se encuentra ubicado sobre la Av. Jorge Newbery, al sur de la Av. de Circunvalación Mario Bravo (Figura 1). Según los datos del Censo 2010 (INDEC, 2010) se trata de un barrio de 1.390 habitantes, distribuidos con una densidad poblacional muy baja en un territorio con gran cantidad de terrenos y espacios sin construcción. Zulaica, Ferraro y Vázquez demuestran, "una transformación considerable respecto del proceso de urbanización anterior desarrollado en esa zona, caracterizada por la presencia de numerosos espacios vacantes." (2012, p. 149).

Figura 1. Barrio Parque Independencia, Mar del Plata (2017)

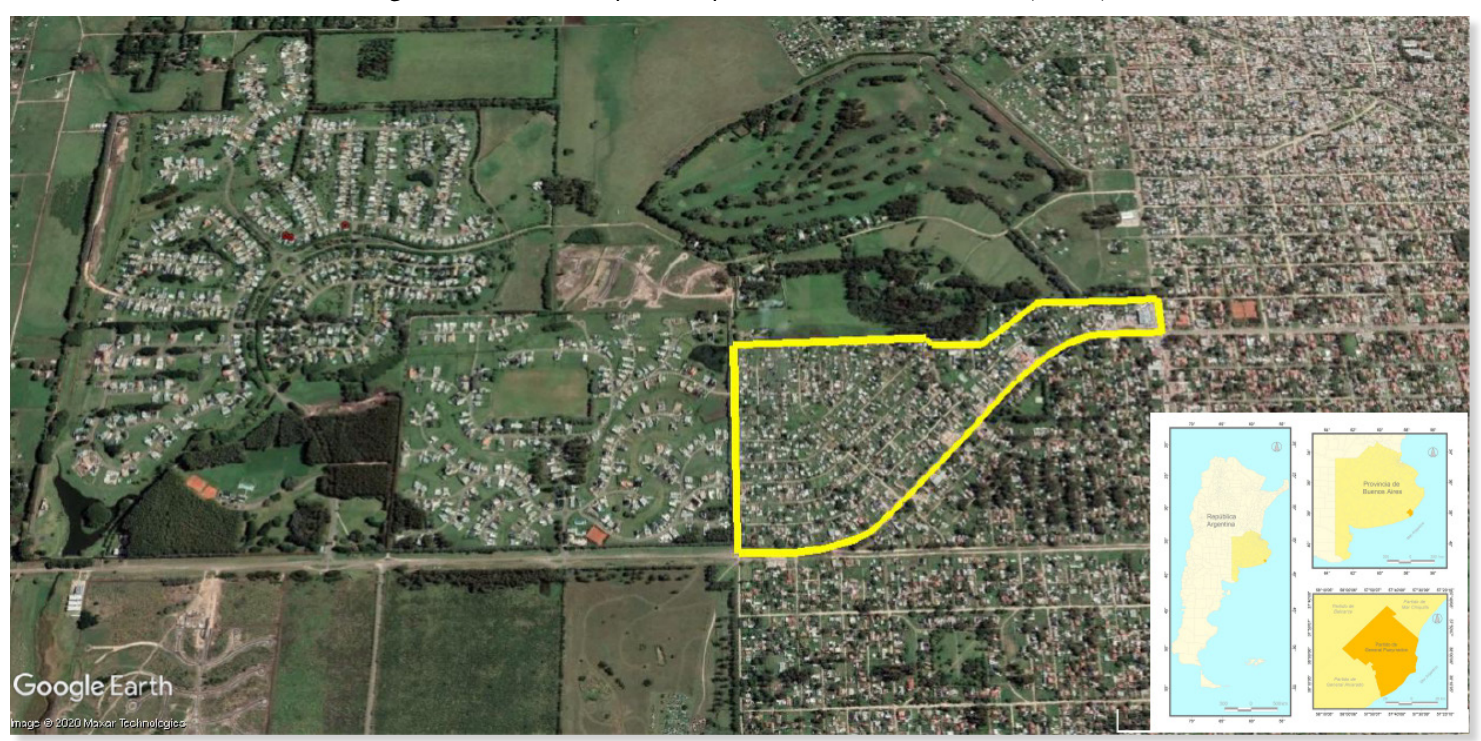

Fuente: elaboración personal con base en Google Earth (2018) 
Concretamente, se consideró la problemática de riesgo de inundación en el barrio Parque Independencia (Mar del Plata), en el periodo comprendido entre 2017-2018. De hecho, esta investigación se encuentra en diálogo con la propuesta de Mujica (2016), quien encuentra en el análisis continuo de los fenómenos aquí estudiados, una disminución en el milimetraje de las lluvias, pero un aumento en la ocurrencia de precipitaciones más intensas en cortos períodos de tiempo, considerando el lapso 1969-2015 (Gráfico 1). El periodo seleccionado continúa con la tendencia que sostiene la autora, resaltando un incremento de inundaciones.

El área se caracteriza por tener viviendas precarias, de chapa, madera y, en algunos casos, ladrillos y membranas en los techos. Dichas viviendas presentan un alto grado de vulnerabilidad y, según los datos relevados en las encuestas realizadas, no cuentan con la totalidad de los servicios básicos, específicamente ausencia de una conexión a la red de gas natural, un bajo porcentaje de acceso al agua potable por conexión a red pública, inexistencia de una red cloacal, por ejemplo (Gráfico 2).

Gráfico 1. Cantidad de eventos de inundaciones registrados en la ciudad de Mar del Plata durante el periodo 1969-2015

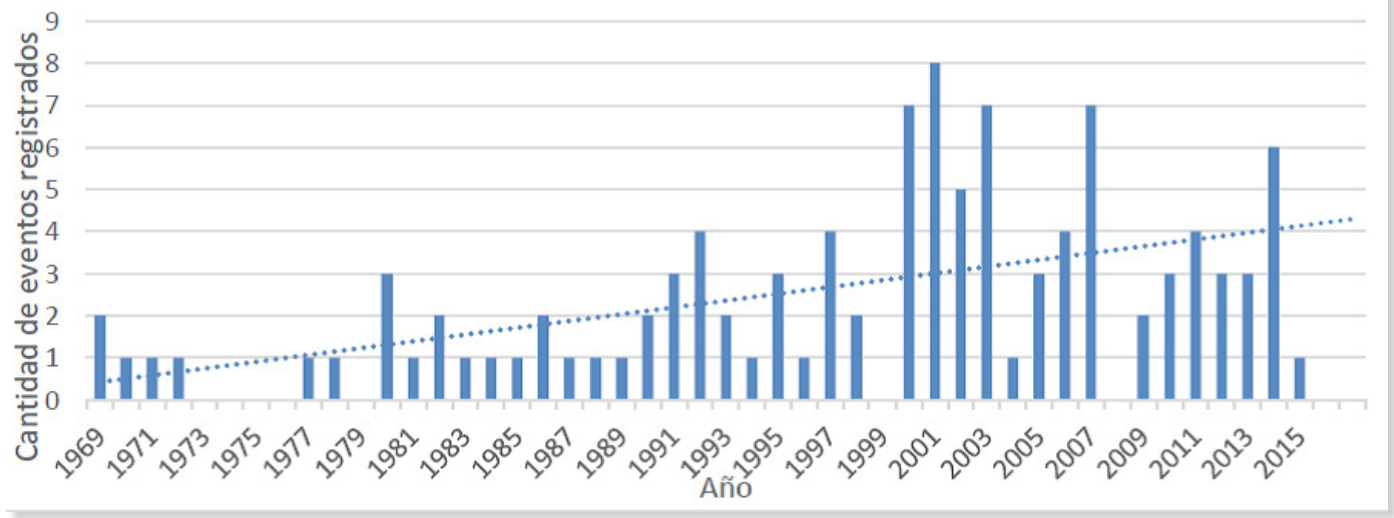

Fuente: Mujica (2016)

Gráfico 2. Características de las viviendas de barrio Parque Independencia (Mar del Plata)

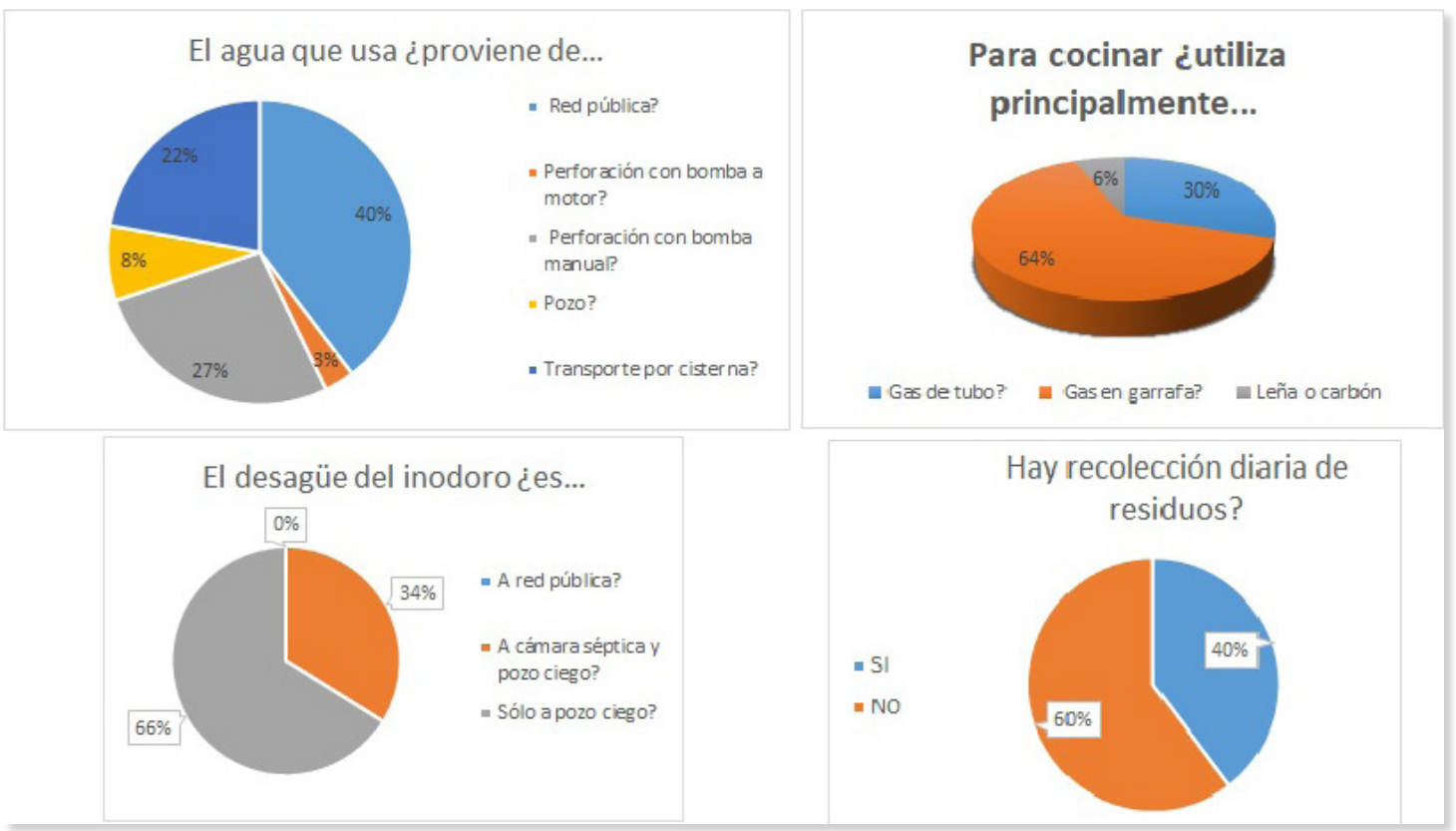

Fuente: elaboración personal con base en relevamiento de encuestas cerradas 
La mayoría de las calles están cubiertas de tierra y granza. En épocas de frecuentes precipitaciones, sobre todo durante la época estival (Tabla 1), se dificulta el traslado de los vecinos debido a continuas anegaciones e inundaciones. Se trata de un asentamiento alejado del centro de la ciudad, frecuentado solo por algunas líneas de transporte público que posibilitan su conexión con el resto de la urbe. Incluso, las unidades de transporte público solo transitan por la avenida Jorge Newbery, y por avenida Mario Bravo, motivo por el cual condiciona la movilidad de sus vecinos en momentos de inclemencias climáticas.

Las urbanizaciones cerradas de Rumencó, Arenas del Sur, Las Prunas y Arenas Chico se encuentran contiguas al área de estudio y sus superficies oscilan entre las 14 y 150 hectáreas ${ }^{1}$. El aumento de emprendimientos urbanos cerrados en la ciudad de Mar del Plata es un proceso que acompaña las lógicas que se desarrollan en diversas áreas metropolitanas del país y la región. Sin embargo, a nivel local, el asentamiento de estos es un fenómeno relativamente reciente. En la ciudad existían antecedentes de urbanizaciones con cerramiento perimetral -por ejemplo, los emprendimientos Lomas del Golf (1965) y Complejo Habitacional SOIP (1974)- caracterizadas por el acceso indiferente y sin control estricto de personas y vehículos. Sin embargo, Mar del Plata no contaba con barrios cerrados

1 Información disponible en: http://www.rumenco.com/; http://www.lasprunas.com/ (consulta junio de 2019). en el sentido que lo expresa o define la Ley Provincial vigente ${ }^{2}$. Si bien cuentan con el amparo jurídico-legal del gobierno municipal y provincial para su emplazamiento, faltaron políticas de gestión sustentables que contemplaran el impacto socioambiental del accionar humano. En la Figura 2, se pueden observar las delimitaciones precisas que existen entre las urbanizaciones privadas anteriormente descriptas (color amarillo) y el barrio vecino a ellos, Parque Independencia (color rojo).

El trabajo con encuestas ha permitido identificar que el barrio está integrado por emigrantes de distintos puntos del país, provenientes del conurbano bonaerense, Misiones, Corrientes, Santiago del Estero, por citar algunos de los lugares de procedencia. Según Lucas Gavilán, un vecino de la zona objeto de estudio, gran parte de los vecinos tiene como anhelo "tener un techo propio y vivir dignamente. Mar del Plata nos da la oportunidad de trabajar y tener nuestro pedazo de tierra". Los terrenos fueron ocupados espontáneamente sin contar con la documentación pertinente para realizar el traspaso de dominio. La obtención de los lotes de esta manera tiene sus consecuencias, ya que se presentan innumerables complicaciones al momento de solicitar algún tipo de asistencia pública al municipio o alguna prestación a las empresas privadas de servicios.

2 El Decreto Ley 27/98 de la provincia de Buenos Aires, en su artículo 1, denomina barrio cerrado "a todo emprendimiento urbanístico destinado a uso residencial predominante con equipamiento comunitario cuyo perímetro podrá materializarse mediante cerramiento".

Tabla 1. Datos del clima en Mar del Plata, Buenos Aires (1981-2010)

\begin{tabular}{|c|c|c|c|c|c|c|c|c|c|c|c|c|}
\hline Valor Medio de & Ene & Feb & Mar & Abr & May & Jun & Jul & Ago & Sep & Oct & Nov & Dic \\
\hline Temperatura $\left({ }^{\circ} \mathrm{C}\right)$ & 20.4 & 19.8 & 18.2 & 14.5 & 11.2 & 8.4 & 7.5 & 8.8 & 10.4 & 13.3 & 15.9 & 18.5 \\
\hline Humedad relativa (\%) & 74.4 & 76.9 & 79.3 & 80.5 & 82.4 & 82.7 & 82.5 & 80.8 & 79.6 & 78.4 & 76.5 & 74.6 \\
\hline Precipitación (mm) & 94.9 & 93.9 & 95.7 & 85.5 & 66.4 & 57.4 & 55.7 & 55.9 & 57.3 & 89.3 & 81.3 & 92.8 \\
\hline $\begin{array}{l}\text { Frecuencia de días } \\
\text { con Precipitación } \\
\text { superior a } 0.1 \mathrm{~mm}\end{array}$ & 8.3 & 7.9 & 9.0 & 9.1 & 8.6 & 8.5 & 8.8 & 7.9 & 7.9 & 9.8 & 9.6 & 8.8 \\
\hline
\end{tabular}

Fuente: Servicio Meteorológico Nacional (2018) 
Figura 2. Área de estudio. Urbanizaciones privadas que limitan con el Barrio Parque Independencia

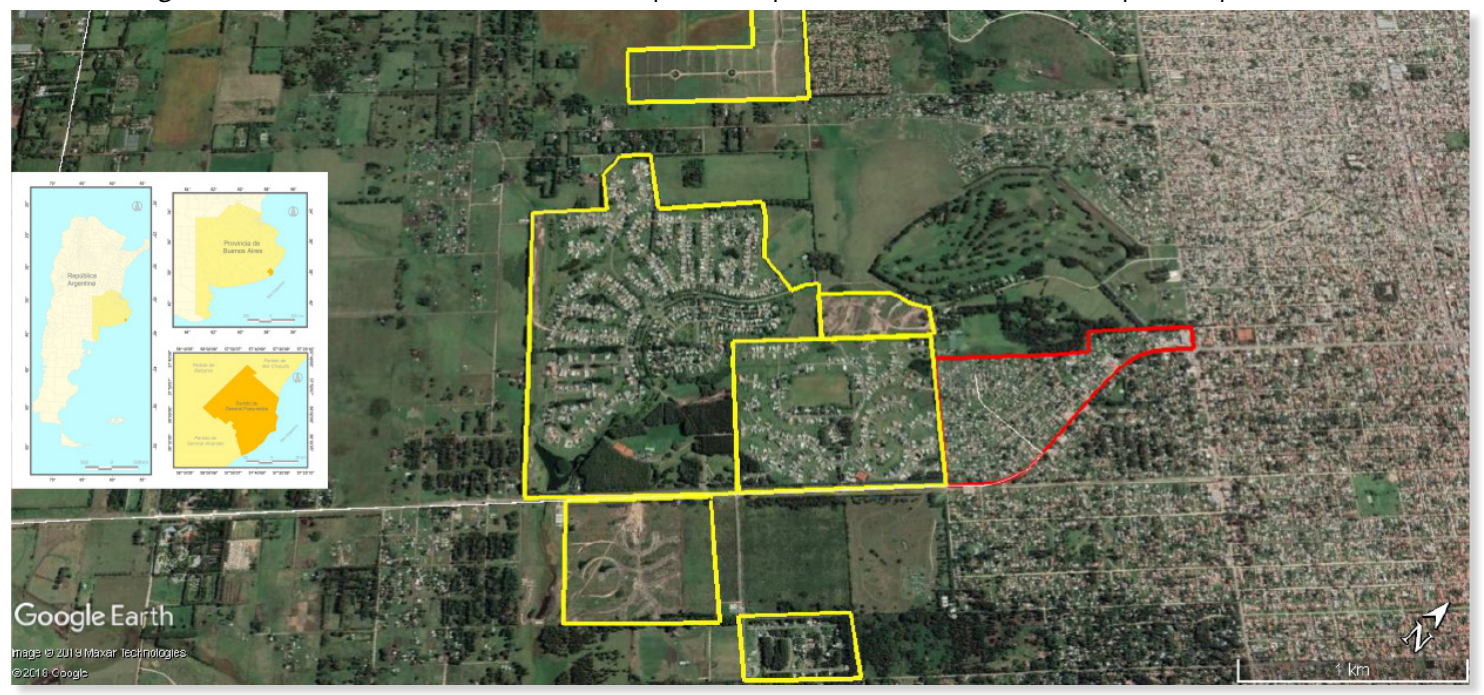

Fuente: elaboración personal con base en Google Earth (2018). Mapas de localización del área en distintas escalas elaborados por: Grupo de Estudios de Población y Territorio (GESPYT, 2010)

El entorno físico natural se caracteriza por la presencia de una forestación escasa, donde predominan especies exóticas como eucaliptos, pinos, cedros, cipreses, acacias, álamos, sauces, ligustros, olmos, fresnos, entre otras. La zona se encuentra atravesada por el arroyo Corrientes, fuente de abastecimiento de agua para animales de corral, rodeado de juncales y pajonales de espadaña ${ }^{3}$. En cercanía al área de estudio se encuentra un tramo de dicho curso de agua.

El relieve de la ciudad de Mar del Plata presenta levantamiento tectónico producto de la acción de fallas de alto ángulo. Este fenómeno le confiere su aspecto de bloques elevados separados por amplios valles y abras espaciosos. De hecho, Mar del Plata está edificada sobre una topografía de bloques altos, llamados pilares tectónicos que raramente superan los 40 metros sobre el nivel del mar, y bloques hundidos, conocidos como fosas tectónicas. En este

\footnotetext{
3 Información disponible en: https://www.mardelplata.gob. ar/Contenido/flora (Fecha de última consulta: Junio de 2019).
}

marco, el área objeto de estudio está localizada sobre un bloque elevado de terreno con una leve depresión que no permite una escorrentía aceptable. La Figura 3 permite observar con mayor detalle la topografía del terreno. Las precipitaciones abundantes entre los meses de octubre y abril de cada año más las características del relieve, provocan situaciones de anegación e inundación. En definitiva: la indiferencia manifiesta del Estado municipal/provincial en torno a la proyección de obras de mejora o infraestructura unida a la modificación producida por el relleno y posterior elevación del terreno producto de los barrios privados, generaron un escenario de riesgo para los habitantes del barrio Parque Independencia. Cardona (1991) afirma que

su configuración se realiza a partir de la distribución espacial de los efectos potenciales que puede causar un evento de una intensidad definida sobre un área geográfica, de acuerdo con el grado de vulnerabilidad de los elementos que componen el medio expuesto (Cardona, 1991, p. 3) 
Figura 3. Topografía del barrio Parque Independencia, Mar del Plata (2018)

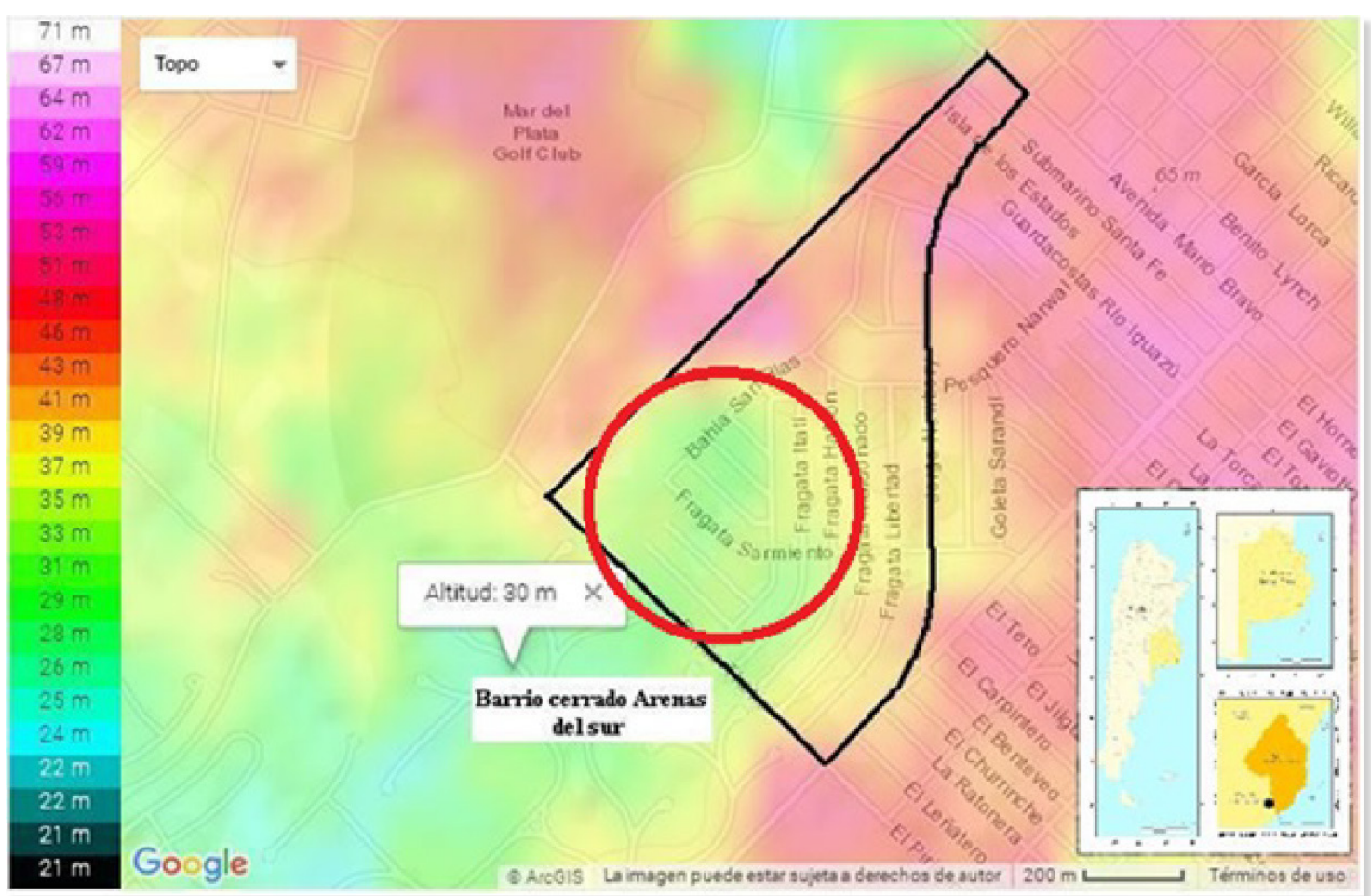

Fuente: Elaboración personal con base en Google Earth (2018)

\section{Resultados}

\section{DIAGNÓSTICO - EVALUACIÓN DE RIESGO}

La observación de la información climática de la ciudad de Mar del Plata en los últimos 30 años (Tabla 1), pone de manifiesto que el comportamiento de las precipitaciones es mayor en los meses que van de octubre a abril de cada año. La probabilidad de ocurrencia de un evento peligroso en el corto plazo es permanente, en especial durante las estaciones de primavera y verano.

Por otra parte, el nivel de exposición y predisposición de los habitantes del barrio Parque Independencia a la pérdida de un elemento o grupo de elementos frente a una amenaza o peligro es fuertemente probable. De hecho, la mayoría de las viviendas están localizadas sobre terrenos en desnivel o con evidentes signos de depresión, característica causada por procesos naturales (relieve) y por acciones humanas (construcción de terraplenes). En tal sentido, entre los factores que convierten al territorio en un escenario de riesgo para los pobladores del barrio destacan: a) la precariedad de las viviendas; b) la acción antrópica (modificaciones del terreno provocadas por agentes privados asociados a las urbanizaciones cerradas); c) la falta de infraestructura que facilite la contención o circulación del agua en periodo de precipitaciones abundantes y; d) las características propias del terreno (bajo nivel para la escorrentía superficial y niveles insuficientes de infiltración, rápida saturación de los suelos). Según la clasificación de fuentes de peligrosidad de sitio, realizada por Monti (2011), nos hallamos ante una tipología natural inducida social o económicamente. Es decir, la

probabilidad de ocurrencia de un fenómeno natural, inducido o potenciado por acciones antrópicas que modifican la dinámica original del mismo, en un sitio y tiempo específico, tal que visualizado desde la perspectiva humana puede producir impactos negativos en la población, la infraestructura y demás bienes, funciones y servicios del ambiente. (Monti, 2011, p. 28)

En el presente caso, dicha situación se debería a la anegación e inundación del barrio Parque Independencia por modificaciones en las pendientes del terreno, las mismas que interfieren en la escorrentía natural del agua. 
La integración de la peligrosidad del evento y la vulnerabilidad de los contextos expuestos resulta necesaria para estimar daño probable de la comunidad ante esta caracterización. Así, a continuación, se establecen las pérdidas probables directas e indirectas que un evento peligroso pudiera llegar a causar en el área de estudio (Tabla 2).

Tabla 2. Estimación del riesgo sobre la base de pérdidas probables directas e indirectas ante un evento de peligro

\begin{tabular}{lll}
\hline \multicolumn{1}{c}{ Evento } & \multicolumn{1}{c}{ Pérdidas probables directas } & \multicolumn{1}{c}{ Pérdidas probables indirectas } \\
\hline & - Pérdida de infraestructura & - Pérdidas de días de clase. \\
& - Cortes de luz. & - Suspensión del transpor- \\
& - Anegamiento de calles & te público de pasajeros. \\
Intensas precipi- & - Deterioro parcial o des- & - Pérdidas de días de trabajo. \\
taciones, provo- & trucción de la vivienda. & - Imposibilidad de ingre- \\
can anegación & - Accidentes viales. & so de asistencia médica. \\
e inundación & - Inundación de terrenos bajos. & - Impedimento de tránsito vehicular. \\
& - Pérdidas de bienes materiales. & - Eventualidad de enfermedades. \\
& - Probabilidad de evacuación, & - Suspensión del servicio de re- \\
& heridas o fallecimiento. & colección de residuos.
\end{tabular}

Fuente: Elaboración personal (2017/18)

\section{CLASIFICACIÓN DE ACTORES SOCIALES}

El relevamiento de información permitió reconocer los actores sociales vinculados con la problemática de riesgos de inundación en el barrio Parque Independencia, con el objetivo de comprender las lógicas e intereses que rodean a cada uno de los involucrados. En la Tabla 3, se han agrupado los actores sociales participantes, según criterios de: a) nivel de organización; b) función que cumplen en el proceso de búsqueda de soluciones y; c) nivel de participación según su escala territorial de acción.

Los actores fueron divididos en "Bien Organizados" y "Menos Organizados". Sobre los primeros, $79 \%$ del total, en su mayoría son actores de tipo Estructural (66\%). Dentro de estos, el número mayor se corresponde a sujetos vinculados con organismos de gobierno (estatales) mientras que el porcentaje restante se vincula con sectores empresariales. El rol principal de todos estos actores en la problemática se correspondería, únicamente con los de tipo Estructural, por estar directamente vinculados con la evolución y el desenlace del proceso de gestión. La actuación que realizan dentro de la problemática también los diferencia: los dedicados a la gestión pública son destinatarios de los reclamos realizados por los demás sectores mientras que los actores de gestión privada suelen intervenir aportando soluciones provisorias ante la ausencia de ayuda estatal. Por ejemplo, tal como puede observarse en distintos medios periodísticos donde resaltan que "los corralones de la zona donan de forma voluntaria materiales para la mejora de las calles" (Diario La Capital, 16/05/2017). A su vez, ambos grupos de actores Estructurales y Bien Organizados, poseen representantes en los dos niveles de participación territorial: local (municipal) y provincial. Dentro de los actores "Bien Organizados", se identifica un grupo menos diverso, cuyo rol en la problemática permite su caracterización como Funcional (los medios de comunicación) y mayoritariamente de origen local. Noticias transmitidas en vivo, artículos periodísticos y videos publicados en los medios gráficos, o en las redes sociales, se constituyen como los medios de exposición de las denuncias y visibilización de la problemática. Dicha forma de actuación contribuye tanto a visibilizar el estado de vulnerabilidad de la población como a cumplir el rol fundamental de servir de nexo entre los ámbitos estructurales de ambas categorías 
reflejadas en la Tabla 3.

Los actores sociales "Menos Organizados" son menos abundantes (21\%) que los "Bien Organizados". Este grupo encuentra referencia en la comunidad local, ya fuera en la Sociedad de Fomento del barrio, en los representantes de comedores o en merenderos vecinales. Sin embargo, el grupo expresa un nivel de actividad significante. En la práctica, las estrategias de reclamos más recurrentes en este grupo consisten en filmaciones, cortes de calles, diálogos con la prensa, siempre con el fin de reducir los niveles de vulnerabilidad frente a peligros naturales. Incluso, hay actividades alternativas como la realización de eventos populares, venta de bonos y rifas con la finalidad de recaudar fondos para el arreglo parcial de las calles. De esta manera, los grupos "Menos Organizados" involucraron en sus acciones al nivel municipal de gobierno.

Tabla 3. Clasificación de actores sociales involucrados en las respuestas frente al riesgo de inundación en Parque Independencia

\begin{tabular}{|c|c|c|c|c|c|}
\hline \multirow{2}{*}{$\begin{array}{l}\text { Nivel de organi- } \\
\text { zación (Sorensen } \\
\text { et al., 1992) }\end{array}$} & \multirow{2}{*}{$\begin{array}{l}\text { Rol en la pro- } \\
\text { blemática (Si- } \\
\text { mioni, 2003) }\end{array}$} & \multirow{2}{*}{$\begin{array}{c}\text { Topología de } \\
\text { actores }\end{array}$} & \multicolumn{3}{|c|}{ Niveles de participación (Sorensen et al., 1992) } \\
\hline & & & Locales & Provinciales & Nacionales \\
\hline \multirow{5}{*}{$\begin{array}{l}\text { Actores bien } \\
\text { organizados }\end{array}$} & \multirow[t]{2}{*}{ Estructurales } & $\begin{array}{l}\text { Organismos } \\
\text { de Gobierno }\end{array}$ & $\begin{array}{l}\text { - Gobierno Municipal } \\
\text { - Emvial } \\
\text { - Consejo Deli- } \\
\text { berante MGP } \\
\text { - Delegación Mu- } \\
\text { nicipal Puerto } \\
\text { - OSSE } \\
\text { - Dirección de De- } \\
\text { fensa Civil } \\
\text { - Unidad Sanitaria } \\
\text { "Ingeniero Miconi” }\end{array}$ & $\begin{array}{l}\text { - Vialidad de } \\
\text { la Provincia }\end{array}$ & $\begin{array}{l}\text { No hay } \\
\text { registro }\end{array}$ \\
\hline & & Sector empresarial & $\begin{array}{l}\text { - Empresa de con- } \\
\text { tenedores } \\
\text { - Corralones } \\
\text { - Empresas de } \\
\text { transporte públi- } \\
\text { co de pasajeros }\end{array}$ & $\begin{array}{l}\text { No hay } \\
\text { registro }\end{array}$ & $\begin{array}{l}\text { No hay } \\
\text { registro }\end{array}$ \\
\hline & \multirow[b]{3}{*}{ Funcionales } & Sector Académico & No hay registro & $\begin{array}{l}\text { No hay } \\
\text { registro }\end{array}$ & $\begin{array}{l}\text { No hay } \\
\text { registro }\end{array}$ \\
\hline & & ONG & No hay registro & $\begin{array}{l}\text { No hay } \\
\text { registro }\end{array}$ & $\begin{array}{l}\text { No hay } \\
\text { registro }\end{array}$ \\
\hline & & $\begin{array}{l}\text { Medios de Co- } \\
\text { municación }\end{array}$ & $\begin{array}{l}\text { - Diario “La Capital” } \\
\text { - Diario “Qué” } \\
\text { - Canal } 2 \text { Mar del Plata } \\
\text { - Canal } 8 \text { Mar del Plata } \\
\text { - Radio Brisas }\end{array}$ & $\begin{array}{l}\text { No hay } \\
\text { registro }\end{array}$ & $\begin{array}{l}\text { No hay } \\
\text { registro }\end{array}$ \\
\hline
\end{tabular}




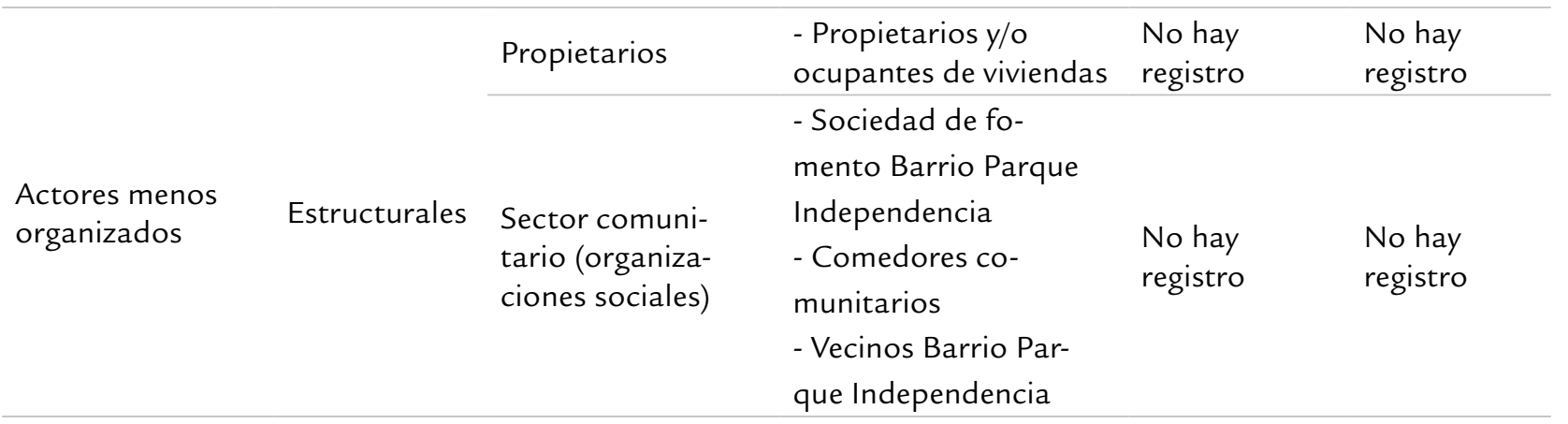

Fuente: elaboración personal (2018)

\section{A MODO DE DISCUSIÓN}

Los habitantes del barrio Parque Independencia han interactuado en forma sostenida $y$ determinada, tanto a nivel individual como colectivo, para reducir los niveles de vulnerabilidad frente a peligros naturales, y han involucrado en sus acciones al nivel municipal de gobierno. No obstante, no se pudieron visibilizar acciones que intenten involucrar al sector académico u otros sectores del gobierno provincial y/o nacional.

La visualización de los reclamos ante la problemática es esperable en escenarios de riesgo que combinen dos condiciones específicas: a) espacios habitables con riesgos a sufrir daños ante la probabilidad de ocurrencia de eventos naturales y agravados por la acción antrópica sobre el terreno $y$; b) una comunidad local automotivada. De no existir una comunidad local con capacidad de lucha, podrían darse resultados muy diferentes, aun tratándose de espacios pequeños y frágiles.

\section{COMENTARIOS FINALES}

Como se deduce del trabajo realizado, las áreas de interfase presentan una gran complejidad debido a que se encuentran sometidas a intensos procesos de cambios (naturales, sociales, económicos) que son relativamente rápidos en el tiempo y que demandan revisiones en las herramientas de análisis tradicional aplicadas al campo y la ciudad.

Los enfoques con perspectiva integral que abarquen distintas miradas disciplinares, alientan nuevas alternativas en la gestión del periurbano. Así, la interpretación del periurbano como un sistema complejo, permite definir subsistemas que resultan apropiados para comprender en profundidad sus elementos, procesos, interacciones, problemas, a fin de dar respuesta a los conflictos emergentes.

En este sentido, la interacción entre los medios social y natural plantea una problemática ambiental compleja en el barrio Parque Independencia. Por una parte, la naturaleza del relieve y la periodicidad de los procesos naturales, está caracterizada por la presencia de precipitaciones durante los periodos comprendidos entre octubre y abril y frecuentes en corto tiempo. Esto, sumado al patrón de ocupación territorial y a las componentes de vulnerabilidad identificadas en los contextos socio-económicos expuestos al peligro, sustenta la caracterización de dicho territorio como un escenario de riesgo.

\section{REFERENCIAS}

Balvanera P. y Cotler, H. (2007). Los servicios ecosistémicos y la toma de decisiones: retos y perspectivas. Gaceta Ecológica, (84-85), 117-123. Número especial.

Cardona, O.D. (1991). Evaluación de la Amenaza,

la Vulnerabilidad y el Riesgo. Taller Regional de Capacitación para la Administración de Desastres ONAD/PNUD/OPS/UNDRO, Bogotá. Cardona, O.D. (1993). Evaluación de la amenaza, la vulnerabilidad y el riesgo. En A. Maskrey 
(Ed.) Los desastres no son naturales. LA RED de estudios sociales, Bogotá.

Cardona, O.D. (2001). La necesidad de repensar de manera holística los conceptos de vulnerabilidad y riesgo: una crítica y una revisión necesaria para la gestión. Internat. work-conference of vulnerability disaster theory and practice. Wegeningen, Amsterdam.

Cendrero, A. y Fischer, D. (1997). Un procedimiento para evaluar la calidad ambiental de las áreas costeras para la planificación y gestión. Journal of Coastal Research, (13), 732-744.

Fernández, R.; Allen, A.; Burmester, M.; Malvares Miguez, M.; Navarro, L.; Olszewski, A. y Sagua, M. (1999). Territorio, Sociedad y Desarrollo Sustentable. Estudios de Sustentabilidad Ambiental Urbana. Buenos Aires: Espacio Editorial - Centro de Investigaciones Ambientales, FAUD, UNMdP. Mar del Plata.

García, R. (2006). Sistemas complejos. Concepto, método y fundamentación epistemológica de la investigación interdisciplinaria. Gedisa Editorial, Barcelona.

Grupo de Estudios de Población y Territorio (2010). Atlas Socio-territorial de Mar del Plata y el Partido de General Pueyrredon. Universidad Nacional de Mar del Plata. Disponible en línea: http://gespyt.blogspot.com/ (consulta noviembre de 2019).

Herzer, H. (1990). Los desastres no son tan naturales como parecen. Medio Ambiente y Urbanización. Buenos Aires, (30), 3-10.

Instituto Nacional de Estadística y Censos (2010). Encuesta permanente de hogares. Buenos Aires.

Lavell, A. (2013). Sobre la Gestión del Riesgo: apuntes hacia una definición. Recuperado de http://www. undp.org/content/dam/undp/documents/cpr/ disred/espanol_/glr_andino/docs/METODOLOGIA\%20DE\%20SISTEMATIZACI\%C3\%93N\%20 PARA\%20DIAGRAMAR/apuntes_hacia_una_ definici_n_de_la_gesti_n_de_riesgo_A_llan_ Lavell.pdf (consulta abril de 2019).

Maskrey, A. (1993). Los desastres no son naturales. LA RED de estudios sociales. Bogotá.

Monti, A. (2007). Dilemas y desafíos de la gestión de riesgos en litorales antropizados de la
Patagonia. Primeras Jornadas de Investigación en Ciencias Sociales. CD ROM. 20pp. UNPSJB. Comodoro Rivadavia.

Monti, A. (2011). La peligrosidad de sitio en escenarios complejos: una propuesta de clasificación integral. Revista Párrafos Geográficos, 10(2), 22-37. Recuperado de http://igeopat.org/parrafosgeograficos/images/RevistasPG/2011_ V10_2/16-2.pdf (consulta junio de 2019).

Monti, A. (2013). Gestión del riesgo y complejidad en el litoral urbanizado de Puerto Madryn. Revista Estudios Ambientales, 1(1), 27-43.

Mujica, C. (2016). Servicios Ambientales. Regulación de Inundaciones en Mar Del Plata (Partido De General Pueyrredón) durante el período 1969/2015. Tesis de grado. Universidad Nacional del Centro de la provincia de Buenos Aires. Tandil.

Saurí Puyol, D. (2003). Tendencias recientes en el análisis geográfico de los riesgos ambientales. AREAS Revista de Ciencias Sociales, (23), 17-30.

Servicio Meteorológico Nacional (2018). Estadísticas climatológicas normales, período 1981-2010. Recuperado de https://www.smn.gob.ar/descarga-de-datos (consulta junio de 2019).

Simioni, D. (2003). La metodología del proyecto Fortalecimiento de la conciencia ciudadana para la formulación de políticas de control de la contaminación atmosférica en tres metrópolis de América Latina: México, D. F., Santiago de Chile y São Paulo. Simioni (Comp.) Contaminación atmosférica y conciencia ciudadana. Comisión Económica para América Latina y el Caribe. Santiago de Chile.

Sorensen, J.C.; Mc Creary, S.T. y Brandani, A. (1992). Costas: arreglos institucionales para manejar ambientes y recursos costeros. US Agency for Internacional development. Internacional Coastal Resources Center. Univ. of Rhode Island, Kingston.

Wilches-Chaux, G. (1993). La vulnerabilidad global. En A. Maskrey (Ed.) Los desastres no son naturales. LA RED de estudios sociales. Primera edición. Bogotá.

Zulaica, L.; Ferraro, R. y Echechuri, H. (2010). El periurbano de Mar del Plata: caracterización 
y perspectivas de abordaje. Primer Simposio de Estudios sobre Ciudad y Territorio. Facultad de Humanidades y Ciencias Sociales. Universidad Nacional de Misiones, Posadas.

Zulaica, L.; Ferraro, R. y Vázquez, P. (2012). Análisis integrado del periurbano de la ciudad

\section{OTRAS FUENTES}

Canal 2 (2017). Inundados en Parque Independencia. Recuperado de https://canal2mdp.com. ar/2017/04/18/18-04-2017-barrio-parque-independencia/ (consulta junio de 2019).

Cadena 3 (2017). El fuerte temporal en Mar del Plata dejó 140 evacuados. Recuperado de https:// www.cadena3.com/noticias/sociedad/fuerte-temporal-mar-del-plata-dejo-140-evacuados_21390 (consulta junio de 2019).

Diario La Capital (16/05/2017). Vecinos de Parque Independencia arreglan con fondos propios las calles del barrio. Recuperado de https://www.lacapitalmdp.com/vecinos-de-parque-independencia-arreglan-con-fondos-propios-las-calles-del-barrio/ (consulta junio de 2019).

Diario Qué (2017). Barro y mucha desidia: así se vive en Parque Independencia. Recuperado de http:// quedigital.com.ar/sociedad/barro-y-mucha-desidia-asi-se-vive-en-parque-independencia/ (consulta junio de 2019).

Ministerio de Gobierno y Justicia (7 de enero 2019). Decreto Ley $N^{\circ} 27 / 98$. Buenos Aires, Argentina.

Municipalidad de General Pueyrredón. Recuperado de https://www.mardelplata.gob.ar/ (consulta junio de 2019).

Paula V. (2017). Temporal e inundaciones en Parque Independencia. Recuperado de https://www. youtube.com/watch?v=Dwr6NTnpTqk\&t=27s (consulta junio de 2019). de Mar del Plata a partir de la identificación y caracterización de unidades ambientales. IX Jornadas Nacionales de Geografía Física. Bahía Blanca. Recuperado de http://www.biblioteca.unlpam.edu.ar/pubpdf/huellas/n14a04zulaica.pdf (consulta septiembre de 2019).

Paula V. (2017). Temporal e inundaciones en Mar del Plata, Parque Independencia. Recuperado de https://www.youtube.com/watch?v=9U5MWICs9NA (consulta junio de 2019).

Radio Brisas (2017). Corte de vecinos del barrio Parque Independencia en Jorge Newbery. Recuperado de https://www.youtube.com/watch?v=cPFZdXIXreQ (consulta junio de 2019).

Resistiendo con aguante (2017). Inundados Parque Independencia - Mar del Plata. Recuperado de https://www.youtube.com/watch?v=9tZYRZwyTjk (consulta junio de 2019).

Teleocho Informa - Canal 8 Mar del Plata (2017). Barrio Parque Independencia: intransitable. Recuperado de https://www.youtube. com/watch?v=0ZriUkL_vHY (consulta junio de 2019).

Teleocho Informa - Canal 8 Mar del Plata (2017). Reclaman por arreglo de calles en barrio Parque Independencia. Recuperado de https://www.youtube.com/watch?v=47D9L5K_wEo\&t=22s (consulta junio de 2019).

Web Barrio Privado Las Prunas. Recuperado de http//www.lasprunas.com/

Web Barrio privado Rumencó. Recuperado de http://www.rumenco.com/

Zona 54 (09/04/2017). Mar del Plata: temporal, inundacionesyevacuados. Recuperado de https://www. zona54.com/noticias/local/1017-mar-del-plata-temporal-inundaciones-y-evacuados.html

\footnotetext{
Mario Alejandro Maya es Profesor de Geografía por la Universidad Nacional de Mar del Plata. Becario doctoral en el Consejo Nacional de Investigaciones Científicas y Técnicas (CONICET). Doctorando en Geografía por la Universidad Nacional del Sur. Desde 2016, es Miembro del "Grupo de Estudios de Ordenación Territorial”, radicado en el Centro de Investigaciones Geográficas y Socio-Ambientales, de la Facultad de Humanidades de la Universidad Nacional de Mar del Plata. Funes 3350, (7600) Mar del Plata, Buenos Aires, Argentina, correo electrónico: mayamarioalejandro@gmail.com
} 